\title{
Anisotropy versus circular dichroism in second harmonic generation from fourfold symmetric arrays of G-shaped nanostructures
}

\author{
E. A. Mamonov, ${ }^{1, *}$ I. A. Kolmychek,,${ }^{1}$ S. Vandendriessche, ${ }^{2}$ M. Hojeij, ${ }^{3}$ Y. Ekinci, ${ }^{3}$ V. K. Valev, ${ }^{4}$ \\ T. Verbiest, ${ }^{2}$ and T. V. Murzina ${ }^{1}$ \\ ${ }^{1}$ Department of Physics, Moscow State University, 119991, Moscow, Russia \\ ${ }^{2}$ Laboratory for Molecular Electronics and Photonics, KU Leuven, Celestijnenlaan 200 D, B-3001 Leuven, Belgium \\ ${ }^{3}$ Laboratory for Micro and Nanotechnology, Paul Scherrer Institute, 5232 Villigen-PSI, Switzerland \\ ${ }^{4}$ NanoPhotonics Centre, Cavendish Laboratory, University of Cambridge, CB3 OHE, Cambridge, United Kingdom
}

(Received 14 September 2013; revised manuscript received 11 February 2014; published 26 March 2014)

\begin{abstract}
Circular dichroism in optical second harmonic generation $(C D-\mathrm{SHG})$ is studied in planar symmetrical arrays of G-shaped and mirror-G-shaped nanostructures. Anisotropic $C D$-SHG measurements demonstrate a strong dependence of the value and the sign of the $C D$ effect on the angle of incidence of the fundamental radiation. We show that both dipole and higher order multipole components of the second order susceptibility are responsible for the $C D$ response from $\mathrm{G}$-shaped nanostructures.
\end{abstract}

DOI: 10.1103/PhysRevB.89.121113

PACS number(s): 42.65.Ky, 78.67.Pt

In recent years, nanostructures have allowed the study of an increasing number of optical phenomena that are rare, weak, or have not been previously observed in nature [1-3]. There is considerable interest in planar metallic chiral nanostructures where the shape of nanoelements and their mutual arrangement govern their plasmonic properties [4,5]. In three dimensions, chirality appears due to the helicity of a 3D object and leads to optical activity, which is an intrinsic feature of many biological structures. 3D chirality is also the mechanism underlying the circular dichroism $(C D)$ effect (different absorption of the left or right circularly polarized light). In a quasi-two-dimensional case, chirality implies the lack of in-plane mirror symmetry of a structure along with breaking the inversion symmetry in the perpendicular direction [6]. The handedness of chiral nanostructures can lead to the $C D$ effect also in the twodimensional case. This effect for the transmitted radiation was theoretically predicted for chiral split-ring resonators [7], planar metal nanostructures consisting of twisted elements [8], sandwichlike metal-dielectric-metal gammadions $[9,10]$, and others. Moreover, circular dichroism transmission spectra were studied theoretically and experimentally in arrays of chiral magnetic nanostructures [11]. It was shown that the combination of magneto-optical properties and of shape chirality modifies further the optical response of such structures. It was also understood that an extrinsic chirality can be introduced even for non-chiral mirror-symmetrical 2D structures [12] for an oblique incidence of the fundamental beam. These linear optical studies encouraged the search for their nonlinear optical counterparts where similar effects can be much more pronounced [13].

Among the nonlinear optical methods, optical second harmonic generation (SHG) is known for its high sensitivity and noninvasive character in diagnostics of surfaces, interfaces, and nanostructures $[14,15]$. In the case of planar metal metamaterials, the SHG response reflects the spatial distribution of local optical fields, which is induced by the shape anisotropy and chirality of nanoelements, as well as their arrangement within an array [16]. It was already demonstrated that the

*mamonov@shg.ru handedness of the samples composed of chiral elements can be determined from a combined analysis of the polarization state and anisotropy of the second harmonic radiation [17,18]. For chiral metamaterials, the nonlinear optical response originates from specific chiral components of the quadratic susceptibility tensor that inverse their sign for the mirror-symmetrical structures [19,20]. These same components also led to an appearance of the circular dichroism effect in SHG that was demonstrated to exceed significantly the similar linear-optical effect in the case of G-shaped nanostructures [4].

Circular dichroism in SHG in chiral molecules was studied in a number of papers [21-24]. These studies reveal high SHG sensitivity to the shape chirality of nanoobjects, which is of particular interest for biological applications [25,26]. A phenomenological model describing the structural and molecular origins of $C D$-SHG was developed [27]. The $C D$ SHG effect was found in nonchiral materials [28] as well due to the interference between the electric dipole and the quadrupole contributions to the nonlinear polarization, while in that case the effect is intrinsically related to the anisotropy of the nonlinear response of a particular structure. Importantly, in the case of achiral nanoobjects the $C D$-SHG effect vanishes after being averaged over all the azimuthal orientations of the structure, while it remains nonzero for chiral structures [4]. Both anisotropic and chiral SHG contributions can change when changing the experimental conditions, e.g., the angle of incidence and polarization of the fundamental radiation, and their careful distinguishing is an important question. Up to now there have been no direct experiments on the role of intrinsic shape anisotropy of chiral nanostructures in the appearance of circular dichroism of their nonlinear optical response, even for their centrosymmetric package within an array. In this paper we study an interplay between the SHG circular dichroism and the SHG azimuthal anisotropy in symmetrically arranged G-shaped nanostructures. We show the importance of the geometry of interaction, especially of the angle of incidence of the fundamental radiation, in the appearance of these effects.

Two samples of regular planar arrays of gold G-shaped and mirror-G-shaped nanostructures were studied. Figure 1(a) shows schematically the structural unit cells, which consist of four $\mathrm{G}$ (or mirror-G) elements, each of them rotated to $90^{\circ}$ with 


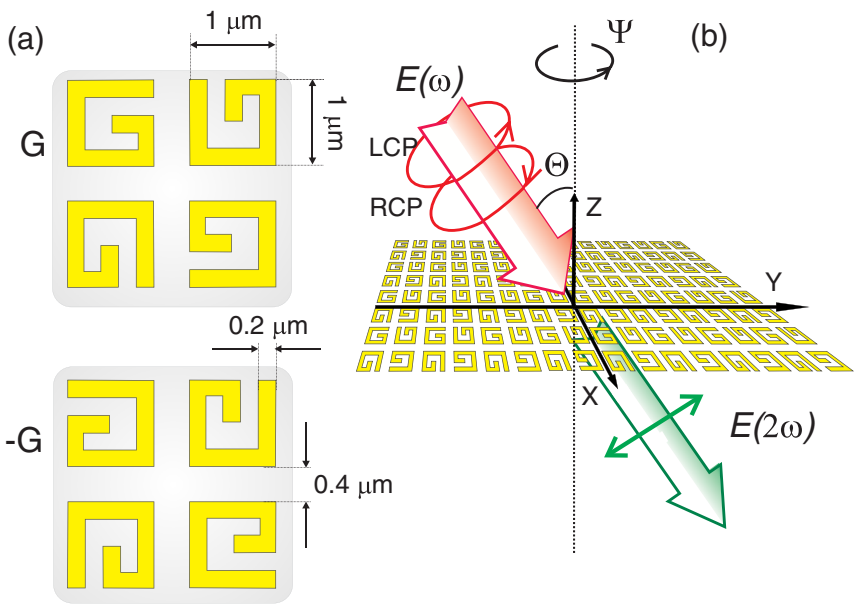

FIG. 1. (Color online) (a) Schemes of elementary cells of symmetric planar G-shaped and mirror-G-shaped structures; (b) scheme of the SHG experiment.

respect to its neighbors. Thus the two studied enantiomorphs are fourfold symmetric in the plane of the structure. The samples are fabricated by electron beam lithography on a glass substrate. An individual G (or mirror-G) element is $1 \mu \mathrm{m}$ in lateral size, $25 \mathrm{~nm}$ thick, and the line of the $\mathrm{G}$ element is $200 \mathrm{~nm}$ wide; the distance between two elements is $400 \mathrm{~nm}$.

For the SHG experiments a Ti:sapphire laser at a wavelength of $795 \mathrm{~nm}$, pulse width of $100 \mathrm{fs}$, and average intensity of $40 \mathrm{~mW}$ was used as a pump radiation [Fig. 1(b)]. The fundamental beam was focused on the sample into a spot of $50 \mu \mathrm{m}$ in diameter, thus over $1200 \mathrm{G}$-shaped elements were irradiated. We analyzed the second harmonic radiation generated in transmission through the sample at different angles of incidence of the fundamental beam. Linearly polarized SHG was spectrally selected by a set of color filters and detected by a photomultiplier. The polarization of the fundamental radiation was controlled by a quarter-wavelength plate. Azimuthal anisotropy of the SHG intensity and of the $C D$ effect were studied as the azimuthal rotation of the sample to $360^{\circ}$ was performed.

Figure 2 shows the dependencies of the $p$-polarized SHG intensity transmitted through the array of G-shaped nanostructures on the azimuthal angle of the sample (plotted on the $y$ axis) and on the angle of the quarter-wavelength plate (plotted on the $x$ axis). The latter controls the polarization state of the fundamental beam. The azimuthal orientations of the quarter-wavelength plate of $0^{\circ}, 90^{\circ}, 180^{\circ}$, and $270^{\circ}$ correspond to the linearly $p$-polarized fundamental beam, while right and left circular polarizations were attained for the angles $45^{\circ}$ and $225^{\circ}$ (right) and $135^{\circ}$ and $315^{\circ}$ (left). The zero azimuthal angle of the sample corresponds to the case as the polarization plane of the fundamental beam is parallel to the side of the $\mathrm{G}$ elements. The measurements are performed for the angle of incidence of $\theta=10^{\circ}$.

It stems from the SHG polarization-anisotropy map shown in Fig. 2 that for all polarizations and for an oblique incidence of the fundamental beam, the SHG intensity reveals a fourfold anisotropy versus the azimuthal sample angle (vertical scale) that is consistent with the symmetry of the arrays of G-shaped

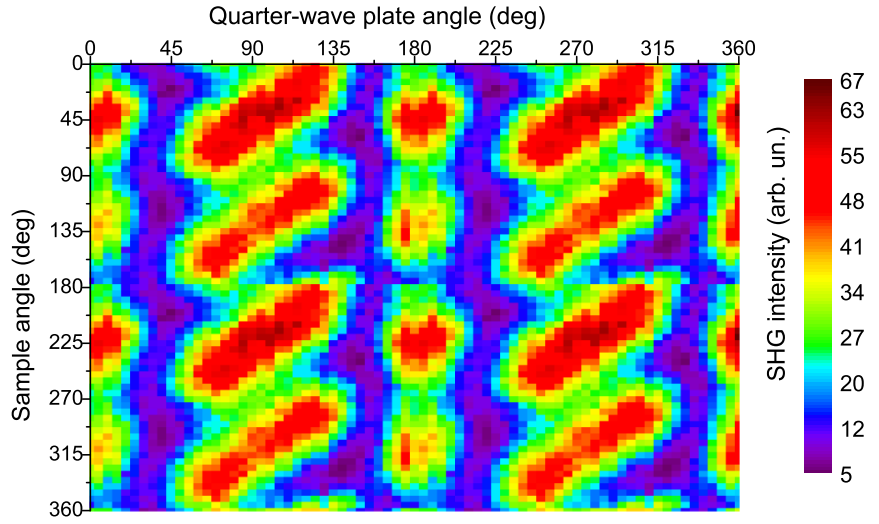

FIG. 2. (Color online) The dependence of the $p$-polarized SHG on the azimuthal angle and on the polarization of the fundamental beam in the array of G-shaped nanostructures. The angles of the quarter-wavelength plate $0^{\circ}, 90^{\circ}, 180^{\circ}$, and $270^{\circ}$ correspond to the linearly $p$-polarized fundamental beam, while right and left circular polarizations are attained for $\alpha=45^{\circ}$ and $\alpha=225^{\circ}$ corresponding to right circular polarization, $\alpha=135^{\circ}$ and $\alpha=315^{\circ}$ to left circular polarization. The angle of incidence is $10^{\circ}$.

nanostructures. The SHG maxima and minima are driven on the azimuthal angle scale depending on the type of the polarization. The most symmetrical azimuthal dependencies with nearly equal amplitudes of the four SHG maxima are observed for circular polarizations of the fundamental beam. Evidently, this anisotropy reveals the fourfold symmetry of the arrays of $\mathrm{G}$ elements.

To study the interplay between the $C D$ and anisotropy effects in the nonlinear optical response, the SHG azimuthal dependencies were measured in the two enantiomorphs for different angles of incidence and for the two circular polarizations of the pump radiation. Figures 3(a) and 3(b) shows such patterns obtained for the $p$-polarized SHG and for $\theta=0^{\circ}$ and $\theta=10^{\circ}$, respectively. Notations $p(s)$ for $\theta=0^{\circ}$ correspond

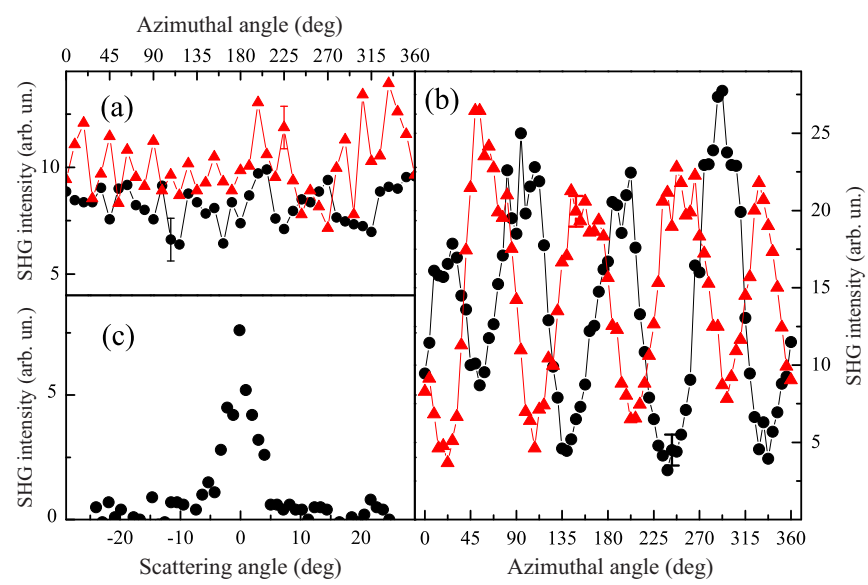

FIG. 3. (Color online) Azimuthal anisotropy patterns of the ppolarized SHG intensity for an array of G-shaped nanostructures obtained for the right (black circles) and left (red triangles) circularly polarized fundamental beam: (a) normal incidence, $\theta=0^{\circ}$ and (b) for $\theta=10^{\circ}$. (c) SHG scattering indicatrix measured for the normal incidence of the circularly polarized fundamental beam. 
to the cases of parallel (perpendicular) orientation of the SHG polarization plane with respect to the side of the $\mathrm{G}$ elements. It can be seen that at normal incidence the SHG azimuthal dependencies are isotropic within the experimental accuracy. On the contrary, fourfold SHG azimuthal patterns are observed at oblique incidence of the pump beam, as can be seen in Fig. 3(b).

Figure 3(c) shows the SHG scattering indicatrix, i.e., the dependence of the SHG intensity on the angle of detection, measured for the circularly polarized fundamental beam. One can see that second harmonic is generated within a narrow angular peak centered around the direction of the specular reflection; the FWHM of approximately $5^{\circ}$ correlates well with the angular aperture of the detection system. The absence of diffusely scattered SHG proves the coherent nature of the SHG response from G-shaped metamaterials.

We studied the $C D$ effect as a function of the angle of incidence of the pump beam. The value of the circular dichroism was calculated as the difference between the SHG intensities attained at different circular polarizations of the fundamental beam and divided to their sum,

$$
C D_{p}=\frac{I_{p}^{\text {right }}-I_{p}^{\text {left }}}{I_{p}^{\text {right }}+I_{p}^{\text {left }}}, \quad C D_{s}=\frac{I_{s}^{\text {right }}-I_{s}^{\text {left }}}{I_{s}^{\text {right }}+I_{s}^{\text {left }}},
$$

where $I_{i}^{j}$ is the intensity of the transmitted SHG, superscripts $j=$ right, left denote the polarization of the fundamental beam, and the subscripts $i=p, s$ correspond to the polarization of the second-order response. In order to avoid the influence of the SHG anisotropy, the averaged SHG intensity was estimated for every measurement by integrating over all the azimuthal orientations of the sample, thus giving the averaged values $\left\langle C D_{p}\right\rangle,\left\langle C D_{s}\right\rangle$. The angle of incidence of the fundamental beam was varied in the interval $\theta=0^{\circ}-40^{\circ}$.

The $\langle C D\rangle$ value versus the angle of incidence estimated for G- and mirror-G-shaped nanostructures are shown in Figs. 4(a) and 4(b). It can be seen that the dependencies are qualitatively similar, while the sign of the circular dichroism is different for

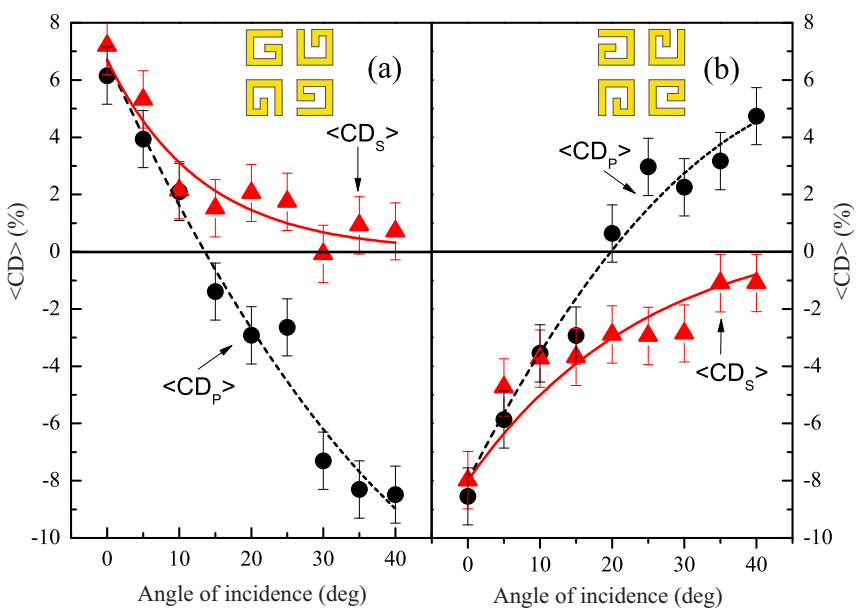

FIG. 4. (Color online) The dependencies of the average circular dichroism on the angle of incidence for the $p$ - (black circles, dashed line) and $s$ - (red triangles, solid line) polarized SHG in the (a) Gshaped and (b) mirror-G-shaped nanomaterials. The lines are guides to the eye.

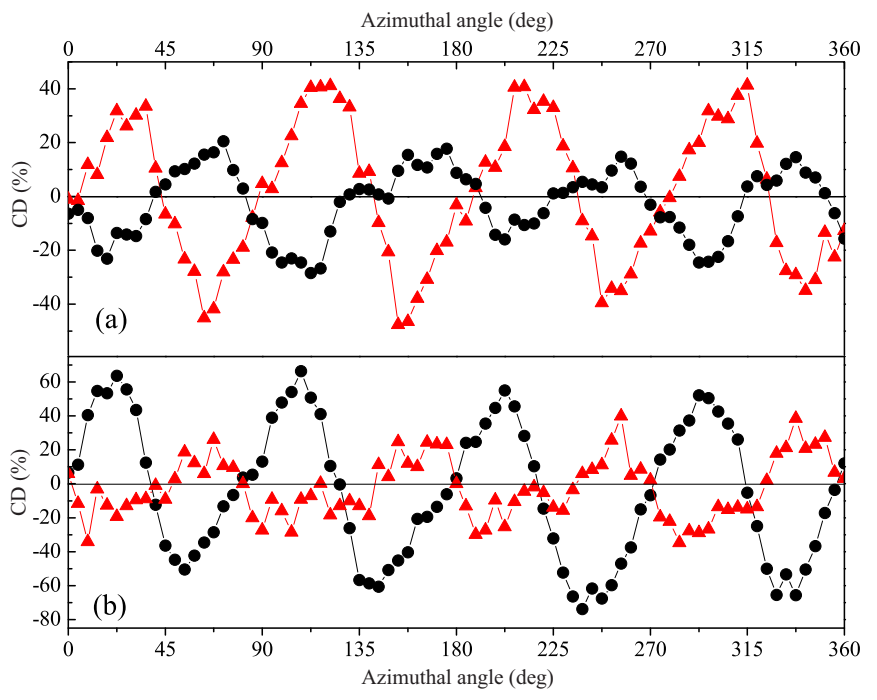

FIG. 5. (Color online) Azimuthal dependencies of the circular dichroism of the $p$ - (black circles) and $s$-polarized (red triangles) SHG intensity at the angle of incidence $15^{\circ}$ in the G-shaped (a) and mirror-G-shaped (b) nanostructures.

the two enantiomorphs. Figure 4(a) shows that for G-shaped structures the $\left\langle C D_{s}\right\rangle$ value decreases nearly to zero with increasing $\theta$, starting from the value of approximately $8 \%$ at normal incidence, while for the $p$-polarized response $\left\langle C D_{p}\right\rangle$ goes to zero and changes the sign at $\theta=15-20^{\circ}$. Mirrorsymmetrical patterns are attained for the mirror-G-shaped structure as can be seen in Fig. 4(b). Thus we demonstrate that the value and the sign of $\langle C D\rangle$ averaged over all the azimuthal orientations of the structure reveal the handedness of the chiral structure under study and are extremely sensitive to the angle of incidence.

Figure 5 shows the $C D$ azimuthal patterns measured for $\mathrm{G}$ and mirror-G-shaped structures at $\theta=15^{\circ}$, i.e., as $\left\langle C D_{p}\right\rangle \approx 0$. Being zero in average, the $C D_{p}$ changes its sign at the azimuthal angles of $\psi=n \cdot \pi / 4, n=0,1,2 \ldots$. Azimuthal $C D_{p}$ and $C D_{s}$ dependencies are shifted in phase with respect to each other, so that the maxima of one of them correspond to the minima of the other. The $C D$ in the maxima of the azimuthal dependencies reaches extremely high values of $40-60 \%$ for the case of the $p$ polarization of the second harmonic. Interestingly, the $C D$ azimuthal dependencies of the two enantiomorphs are mirrorlike images of each other. Thus the $C D$ can reach different values and even change the sign depending on the azimuthal orientation of the sample, which demonstrates the crucial interdependence of anisotropy and chirality.

To explain the observed phenomena, the symmetry of the $\hat{\chi}^{(2)}$ tensor was analyzed for the considered chiral structures. For a fourfold symmetric array of chiral $\mathrm{G}$ nanoelements the following dipole components are nonzero: $\chi_{z z z}, \chi_{z x x}=$ $\chi_{z y y}, \chi_{x x z}=\chi_{y y z}, \chi_{x y z}=-\chi_{y x z}[21,29]$, where the Cartesian coordinate system is chosen as shown in Fig. 1(b), and the permutation symmetry of the two last indices is assumed. Thus the dipole $p$ - and $s$-polarized SHG intensity induced by a circularly-polarized fundamental beam $E_{\omega}=(1 \pm i) E_{0}$ 
(here plus or minus correspond to the right and left directions of circular polarization) can be written as [21]:

$$
\begin{aligned}
I_{p}^{\text {right }, \text { left }} \propto & \sin ^{2} \theta \mid-\chi_{z x x} \sin ^{2} \theta+\chi_{z z z} \sin ^{2} \theta \\
& +2 \chi_{x x z} \cos ^{2} \theta \pm\left. i 2 \chi_{x y z} \cos \theta\right|^{2} E_{0}^{2} . \\
I_{s}^{\text {right }, \text { left }} \propto & \sin ^{2} \theta\left|\chi_{x y z} \cos \theta \pm i \chi_{x x z}\right|^{2} E_{0}^{2} .
\end{aligned}
$$

It stems from Eqs. (2) and (3) that (i) the SHG intensity should be zero at normal incidence of the fundamental beam $(\theta=0)$ for the linearly polarized second harmonic, $I_{p, s}^{r i g h t, l e f t}=0$, and (ii) no azimuthal anisotropy of the electricdipole SHG can be expected at oblique incidence for arbitrary $\theta$ values. This conclusion contradicts with the SHG experimental data described above, which shows the existence of a nonzero coherent SHG at normal incidence [Fig. 3(a)] and a fourfold SHG anisotropy at oblique incidence of the fundamental radiation [Fig. 3(b)]. Thus we have to assume that the description of the SHG in G-shaped isotropic structure by the electric-dipole SHG contribution only is not valid and requires consideration of the higher-order nonlinearities as well. The first candidates here are the magnetic-dipole and electric-quadrupole terms of the second-order polarization [30], which are different in their mechanisms while revealing similar symmetry properties [21]. The symmetry analysis $[21,29]$ shows that these contributions possess the necessary properties that are observed in our experiments.

$C D$-SHG not depending on the sample's azimuthal position can be described in terms of the electric dipole susceptibility components. Now the formulas for the $C D$-SHG as a function of the angle of incidence $\theta$ can be obtained by substituting Eqs. (2) and (3) into Eq. (1). Thus for the case of the $s$-polarized SHG the $C D$ takes the form

$$
\left\langle C D_{s}\right\rangle=2 \cos \theta \frac{\operatorname{Im} \chi_{x y z} \operatorname{Re} \chi_{x x z}-\operatorname{Re} \chi_{x y z} \operatorname{Im} \chi_{x x z}}{\left|\chi_{x x z}\right|^{2}+\left|\chi_{x y z}\right|^{2} \cos ^{2} \theta},
$$

while the analogous expression for the $p$-polarized SHG is more intricate:

$$
\begin{gathered}
\left\langle C D_{p}\right\rangle=\frac{4\left(\operatorname{Im} \chi_{x x z} \operatorname{Re} \chi_{x y z}-\operatorname{Re} \chi_{x x z} \operatorname{Im} \chi_{x y z}\right) \cos ^{3} \theta+2\left(\operatorname{Im}\left(\chi_{z z z}-\chi_{z x x}\right) \operatorname{Re} \chi_{x y z}+\operatorname{Re}\left(\chi_{z z z}-\chi_{z x x}\right) \operatorname{Im} \chi_{x y z}\right) \sin \theta \sin 2 \theta}{4\left|\chi_{x x z}\right|^{2} \cos ^{4} \theta+\left(\left|\chi_{z x x}\right|^{2}+\left|\chi_{z z z}\right|^{2}-2\left(\operatorname{Re} \chi_{z x x} \operatorname{Re} \chi_{z z z}+\operatorname{Im} \chi_{z x x} \operatorname{Im} \chi_{z z z}\right)\right) \sin ^{4} \theta} \\
+4\left(\left|\chi_{x y z}\right|^{2}+\left(\operatorname{Re}\left(\chi_{z x x}-\chi_{z z z}\right) \operatorname{Re} \chi_{x x z}+\operatorname{Im}\left(\chi_{z z z}-\chi_{z x x}\right) \operatorname{Im} \chi_{x x z}\right) \sin ^{2} \theta\right) \cos ^{2} \theta
\end{gathered}
$$

It follows from Eqs. (4) and (5) that $\left\langle C D_{s}\right\rangle(\theta)$ decreases monotonously with the angle of incidence, while $\left\langle C D_{p}\right\rangle(\theta)$ can change its sign at certain $\theta$ values. Moreover, both $\left\langle C D_{p}\right\rangle(\theta)$ and $\left\langle C D_{s}\right\rangle(\theta)$ have opposite signs for the different enantiomorphs, which is determined by the different signs of the chiral dipole component $\chi_{x y z}$ for the case of G-shaped and mirror G-shaped elements. Thus the dependencies in Figs. 4(a) and 4(b) look like mirror images of each other. These conclusions correlate well with the experimental data shown in Fig. 4.

In conclusion, azimuthal dependencies of the circular dichroism effect in second harmonic generation (CD-SHG) are experimentally studied in fourfold symmetric planar periodic arrays of G-shaped and mirror-G-shaped gold nanostructures. We show that $C D$-SHG at normal incidence for the circularly polarized fundamental beam is about $8 \%$ and originates purely from the intrinsic chirality of G-based structures. At oblique angles of incidence, azimuthal $C D-\mathrm{SHG}$ patterns are fourfold symmetric and reveal the appearance of the anisotropy of chiral nanoelements. Importantly, the average $C D$ effect for different enantiomorphs have different signs for any angle of incidence of the fundamental radiation. Thus we demonstrate that the SHG probe in the case of planar metamaterials can serve as an extremely sensitive probe of their symmetry and allows us to distinguish between the chirality and structural anisotropy effects.

This work was partially supported by RFBR Grant Nos. 14-02-00446a and 12-02-31338, by the Federal Program of the Russian Ministry of Education and Science (Grant No. 8393). V.K.V. acknowledges financial support from the EPSRC Grant No. EP/G060649/1. S.V. is grateful for financial support from the FWO-Vlaanderen.
[1] A. Papakostas, A. Potts, D. M. Bagnall, S. L. Prosvirnin, H. J. Coles, and N. I. Zheludev, Phys. Rev. Lett. 90, 107404 (2003).

[2] V. A. Fedotov, P. L. Mladyonov, S. L. Prosvirnin, A. V. Rogacheva, Y. Chen, and N. I. Zheludev, Phys. Rev. Lett. 97, 167401 (2006).

[3] A. S. Schwanecke, A. Krasavin, D. M. Bagnall, A. Potts, A. V. Zayats, and N. I. Zheludev, Phys. Rev. Lett. 91, 247404 (2003).

[4] V. K. Valev, N. Smisdom, A. V. Silhanek, B. De Clercq, W. Gillijns, M. Ameloot, V. V. Moshchalkov, and T. Verbiest, Nano Lett. 9, 3945 (2009).
[5] S. Linden, F. B. P. Niesler, J. Förstner, Y. Grynko, T. Meier, and M. Wegener, Phys. Rev. Lett. 109, 015502 (2012).

[6] M. Kuwata-Gonokami, N. Saito, Y. Ino, M. Kauranen, K. Jefimovs, T. Vallius, J. Turunen, and Y. Svirko, Phys. Rev. Lett. 95, 227401 (2005)

[7] S. V. Zhukovsky, A. V. Novitsky, and V. M. Galynsky, Opt. Lett. 34, 1988 (2009).

[8] V. A. Fedotov, A. S. Schwanecke, N. I. Zheludev, V. V. Khardikov, and S. L. Prosvirnin, Nano Lett. 7, 1996 (2007).

[9] D.-H. Kwon, P. L. Werner, and D. H. Werner, Opt. Express 16, 11802 (2008). 
[10] T. Cao, L. Zhang, R. E. Simpson, C. Wei, and M. J. Cryan, Opt. Express 21, 27841 (2013).

[11] M. Decker, M. W. Klein, M. Wegener, and S. Linden, Opt. Lett. 32, 856 (2007).

[12] E. Plum, V. A. Fedotov, and N. I. Zheludev, J. Opt. A: Pure Appl. Opt. 11, 074009 (2009).

[13] V. K. Valev, J. J. Baumberg, C. Sibilia, and T. Verbiest, Adv. Mater. 25, 2517 (2013).

[14] V. K. Valev, Langmuir 28, 15454 (2012).

[15] M. Kauranen and A. V. Zayats, Nat. Photon. 6, 737 (2012).

[16] V. K. Valev, A. V. Silhanek, N. Smisdom, B. D. Clercq, W. Gillijns, O. A. Aktsipetrov, M. Ameloot, V. V. Moshchalkov, and T. Verbiest, Opt. Express 18, 8286 (2010).

[17] E. A. Mamonov, T. V. Murzina, I. A. Kolmychek, A. I. Maydykovsky, V. K. Valev, A. V. Silhanek, E. Ponizovskaya, A. Bratkovsky, T. Verbiest, V. V. Moshchalkov, and O. A. Aktsipetrov, Opt. Lett. 36, 3681 (2011).

[18] E. Mamonov, T. Murzina, I. Kolmychek, A. Maydykovsky, V. Valev, A. Silhanek, T. Verbiest, V. Moshchalkov, and O. Aktsipetrov, Opt. Express 20, 8518 (2012).

[19] V. K. Valev, A. V. Silhanek, N. Verellen, W. Gillijns, P. Van Dorpe, O. A. Aktsipetrov, G. A. E. Vandenbosch, V. V. Moshchalkov, and T. Verbiest, Phys. Rev. Lett. 104, 127401 (2010).
[20] M. Kauranen, S. V. Elshocht, T. Verbiest, and A. Persoons, J. Chem. Phys. 112, 1497 (2000).

[21] S. Sioncke, T. Verbiest, and A. Persoons, Mater. Sci. Eng., R 42, 115 (2003).

[22] M. Schanne-Klein, F. Hache, T. Brotin, C. Andraud, and A. Collet, Chem. Phys. Lett. 338, 159 (2001).

[23] T. Petralli-Mallow, T. M. Wong, J. D. Byers, H. I. Yee, and J. M. Hicks, J. Phys. Chem. 97, 1383 (1993).

[24] S. Foerier, I. A. Kolmychek, O. A. Aktsipetrov, T. Verbiest, and V. K. Valev, ChemPhysChem 10, 1431 (2009).

[25] X. Chen, C. Raggio, and P. J. Campagnola, Opt. Lett. 37, 3837 (2012).

[26] H. Lee, M. J. Huttunen, K.-J. Hsu, M. Partanen, G.-Y. Zhuo, M. Kauranen, and S.-W. Chu, Biomed. Opt. Express 4, 909 (2013).

[27] G. J. Simpson, J. Chem. Phys. 117, 3398 (2002).

[28] X. Li, J. Willits, S. T. Cundiff, I. M. P. Aarts, A. A. E. Stevens, and D. S. Dessau, Appl. Phys. Lett. 89, 022102 (2006).

[29] O. A. Aktsipetrov, I. M. Baranova, and Yu. A. Il'inskiŭ, Sov. Phys. JETP 64, 167 (1986) [Zh. Eksp. Teor. Fiz. 91, 287 (1986)].

[30] Y. R. Shen, The Principles of Nonlinear Optics (Wiley, New York, 1984). 